Ann. Zootech., I 1962,11 (3), I59-172

\title{
SUPPLÉMENTATION D'UN RÉGIME COMPLEXE PAR LA DL MÉTHIONINE DE SYNTHĖSE, CHEZ LE PORC.
}

\author{
A. RERIT, J. LOUGNON et R. PION \\ Ave la collaberation technique de L. lisgrand et F. IIartivit \\ Station de Recherches sur l'Élevage des Porcins, \\ Station centrale de Recherches de Nutrition, \\ Centre national de Recherches zootechniques, Jouy-en-Josas (Seine-et-Oise)
}

\section{SOMMAIRE}

I. - I 'addition de I, 5 p. I 000 de I)L méthionine à un régime de base complexe (composition : 78 p. Ioo d'urge - + p. Ioo de tourteau d'arachide.- .4 p. Ioo de tourteal de soja - 3 p. 100 de farine de luzerne $-4 \mathrm{p}$. 100 de levure de distillerie $-2 \mathrm{p}$. roo de farine de poisson $-2 \mathrm{p}$. I00 de farine de viande - 3 p. Ioo de mélange minéral et vitaminique) contenant $0,5 \mathrm{p}$. I00 à $0,6 \mathrm{p}$. Ioo dacides aminés soufrés et $0,8 \mathrm{p}$. Ioo de lysine, se traduit par une amélioration de la vitesse de croissance (24 p. I0o) et de l'indice de consommation ( $8 \mathrm{p}$. Ioo) pour les animaux pesant entre 20 et $60 \mathrm{~kg}$. Cette amélioration est réduite chez les animaux de poids supérieur à $60 \mathrm{~kg}$. J'amélioration de croissance peut être attribuée à une consommation journalière accrue et à un meilleur équilibre entre acides aminés. La composition corporelle des animaux n'est pas modifiée, ce qui signifie que les rétentions azotée et calorique ont subi des évolutions semblables.

II. - L'addition de 3 p. 1000 de DL méthionine se traduit par une amélioration beaucoup moins sensible des performances des aninaux (augmentation de 12 p. roo de la vitesse de croissance, diminution de $4 \mathrm{p}$. Ioo de l'indice de consommation) pendant la première période de croissance (20 à $60 \mathrm{~kg}$ ). Tout se passe alors comme si un tel apport de méthionine était une source de déséquilibre.

III. - Compte tenu du fait que les acides aminés soufrés sont les facteurs limitants de ce régime, le besoin en ces acides aminés a pu être estimé. Dans nos conditions expérimentales, leur pourcentage optinum dans le régime est de l'ordre de 0,6 à 0,7 p. Ioo pour les animaux de 20 à $60 \mathrm{~kg}$ et de 0,4 à $0,5 \mathrm{p}$. roo pour les animaux de 60 à $100 \mathrm{~kg}$.

IV. - Liutilisation pour l'alimentation des porcs d'un régime dont le taux azoté répond aux normes recommandées par divers organismes scientifiques (Fédération Européenne de Zootechnie, National Research Council) ne permet pas toujours de couvrir le besoin en certains acides aminés.

\section{INTRODUCTION}

L'obtention, par synthèse ou par extraction, de certains acides aninés à une échelle industrielle et l'abaissement corrélatif de leur prix de revient permet actuellement de reconsidérer le problème des supplémentations en alimentation animale. Aux supplémentations interprotéines utilisées dans la pratique zootechnique, la tendance actuelle est de substituer une supplémentation par un ou plusieurs acides aminés de synthèse. 
Cette tendance se traduit, en aviculture, par l'introduction presque systématique de méthionine dans les régimes, introduction d'autant plus justifiée que le besoin en acides aminés soufrés est particulièrement élevé chez les volailles. Pour le Porc, le problème se pose en termes différents en raison de l'incertitude où l'on est sur le niveau exact du besoin en acides aminés indispensables, malgré les nombreux travaux exécutés à ce sujet (Almovist, I959). L'expérience faisant l'objet du présent article avait pour but de préciser dans quelle mesure un régime à teneur en protéines conforme aux normes recommandées par différents organismes scientifiques (National Research Council, Fédération İuropéenne de Zootechnie) était capable de couvrir le besoin spécifique en aminoacides du Porc en croissance.

Animaux et rigimes :

\section{MATÉRIEL ET MÉTHODES}

Trois lots de 12 pores de race Large White provenant de I 2 portées différentes sont constitués suivant le dispositif des blocs complets (SNEDECOR, I961) ; au sein d'une même portée, trois animaux sont choisis de même sexe et de nềme poids au sevrage et sont répartis dans chacun des trois lots.

TABLE:AU I

Composition du rigime de base (cn p. Iоo)

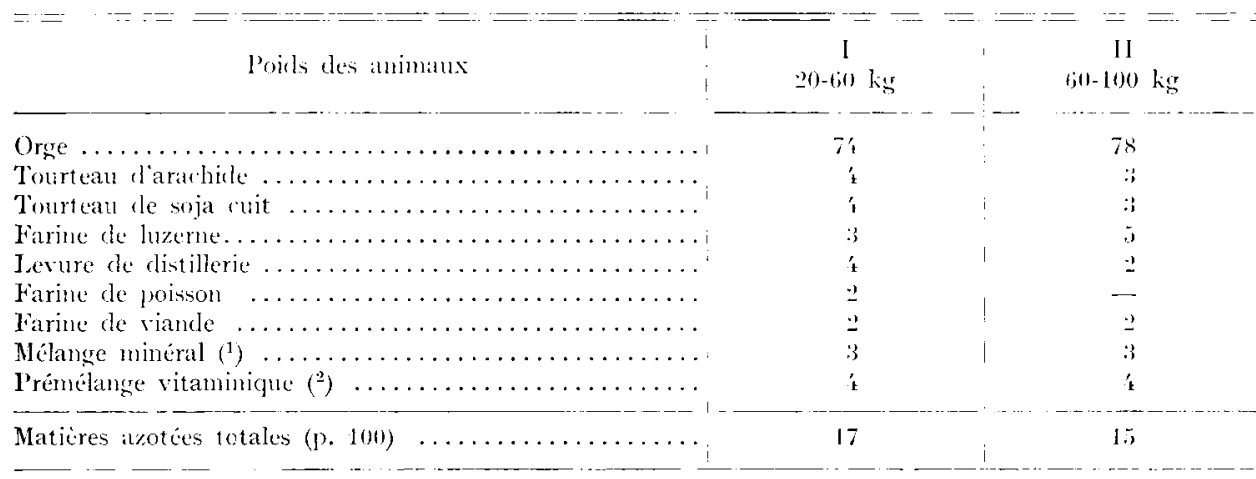

(1) Milange minéral :

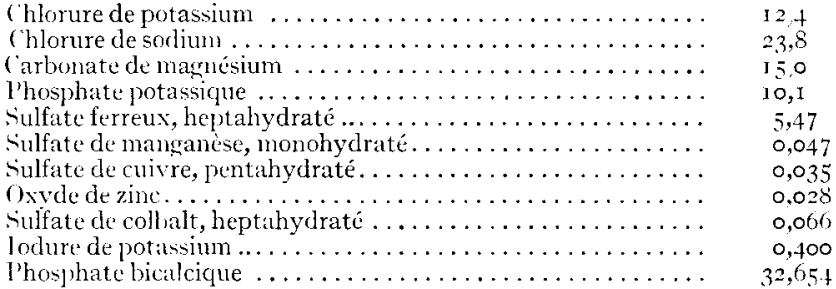

(2) Mélange átaminique ì base d'orge apportant :

Par kg de régime

Vitamine A

Vitamine $\mathrm{D}$

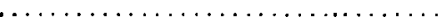

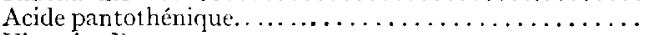

Vitamine $B_{12} \ldots \ldots \ldots \ldots \ldots \ldots \ldots \ldots \ldots \ldots \ldots$

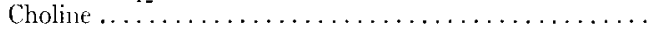

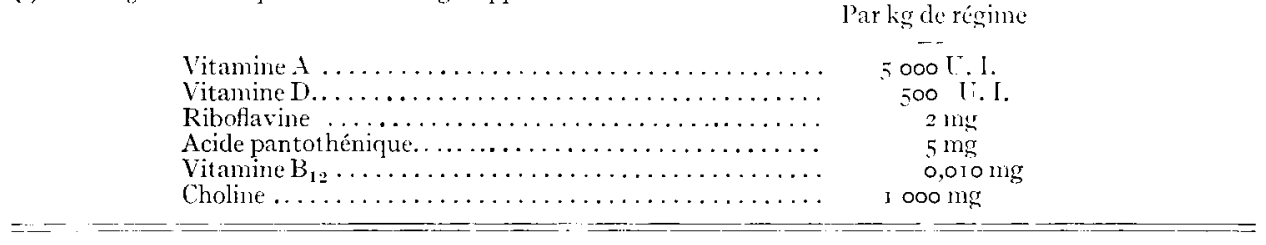


Les régimes distribués en alimentation semi ad libitum entre le sevrage et le poids de roo kg sont les suivants:

- Régime A : régime de base conforme aux " normes " azotées de la lédération Furopéenne de Zootechnie :

- Aliment I : I 7 p. 100 de matières azotées brutes pour les animaux entre 20 et $60 \mathrm{~kg}$.

- Aliment II : I5 p. 100 de matières azotées brutes pour les animaux de poids supérieur à $60 \mathrm{~kg}(1)$ (tableau I).

- Régimes $\mathrm{B}$ et $\mathrm{C}$ : régime de base additionné respectivement de I,5 P. I ooo et 3,0 P. I ooo de DL, méthionine.

Au cours de leur croissance, les animaux sont pesés à intervalles réguliers (tous les i4 jours). Les quantités joumalières de nourriture consonmées sont enregistrées.

Après abattage, diverses mesures sont effectuées sur les carcasses (notamment détermination de l'épaisseur du lard dorsal et du rapport " morceaux maigres " aux " morceaux gras ").

\section{Méthodes analytiques:}

Les techniques qui suivent ont été utilisées pour analyser les régimes :

- Matières azotées totales : méthode de KJELDAIIL.

- Matières cellulosiques : méthode dérivée de la néthode dite de la station agronomique de Weende (IAn EnTwistle et Hunter, i949, Hunter, i948).

- Matières minérales (cendres) : calcination au four à $55^{\circ} \mathrm{C}$ pendant 6 heures.

- Matières grasses : méthode normalisée de l'Institut professionnel de Contrôle et Recherches Scientifiques des Industries de l'Alimentation Animale : extraction simple par l'éther éthylique au soxhlet pendant 4 heures, puis 2 heures, après mélange avec sulfate de sodium anhydre.

- Acides aminées :

- Hydrolyse : on utilise l'acide chlorhydrique $6 \mathrm{~N}$ chauffé à retlux au bain d'huile ( $25^{\circ} \mathrm{C}$ ), ce qui assure une ébullition très douce. $500 \mathrm{ml}$ d'acide sont utilisés pour hydrolyser $200 \mathrm{mg}$ d'aliment. Ies hydrolysats sont cencentrés sous vide à l'évaporateur rotatif

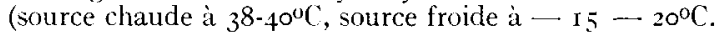

- Séparation et dosage : les acides aminés libérés par hydrolyse acide sont séparés par chromatographie sur colonne selon MoORE et al.(1958). Les fractions obtenues sont analysées par la méthode de Moore et STEIN (1954) en utilisant I $\mathrm{ml}$ de réactif pour des fractions de I, I ml.

-- Méthionine surajoutée : -- extraction selon la méthode de RoIDENBurg et Rosenberg (1956).

- dosage microbiologique (lintillier et al., I954).

L'analyse statistique des résultats est opérée en utilisant le test de Tukfy (Lison, 1958).

\section{RÉSULTATS}

\section{Io Composition chimigue des RÉGIIES}

Flle est rapportée dans le tableau 2. Le tableau 3 concerne la composition en aminoacides ; 1'analyse n'a porté que sur l'aliment utilisé au cours de la deuxième période expérimentale (60-I0o kg de poids vif). Pour chacun des aliments, les teneurs en en acides aminés ont, en outre, été calculées en utilisant soit des résultats d'analyse chromatographique (méthode de MoORE et STEIN) obtenus dans ce laboratoire (orge, arachide, soja, farine de hareng de Norvège, luzerne), soit des données de la littérature, résultats de dosages chromatographiques: farine de viande (EAsToE et LoNG, I960), on microbiologiques : levure de distillerie (Hors et al, I946 $a, b$, I947 $a, b, c$; I948 $a$, $b, c$; I949 $a, b$, - STockes et $a l .$, I945-Irmax et al., I956, I958- Wir.IAMs, I955).

(1) Soit respectivement 140 of a $20 \mathrm{~g}$ de matiers azotés digestibles par unité fourratèn. 
TABLEAU 2

Composition chimique des régimes

\begin{tabular}{|c|c|c|}
\hline & $\begin{array}{l}\text { Animaux de } \\
20 \text { à } 60 \mathrm{~kg}\end{array}$ & $\begin{array}{l}\text { Animaux de } \\
\text { cio à } 100 \mathrm{~kg}\end{array}$ \\
\hline Humidité .... & 12,7 & 12,8 \\
\hline Matière sèche .... & 87,3 & 87,2 \\
\hline Matières minérales $\ldots$. & 5,9 & 4,8 \\
\hline Matières cellulosiques $\ldots \ldots \ldots \ldots \ldots \ldots \ldots \ldots \ldots \ldots \ldots$ & 5,1 & 5,6 \\
\hline Matières azotées totales $(N \times 6,2,25) \ldots \ldots \ldots \ldots \ldots \ldots$ & 17,6 & 15,1 \\
\hline 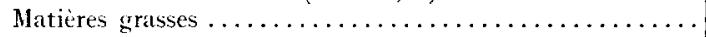 & 5,3 & 2,1 \\
\hline 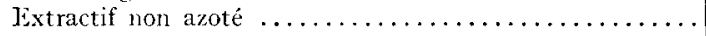 & 53,3 & 59,6 \\
\hline Unités fourragères pour $100 \mathrm{~kg} \ldots \ldots \ldots \ldots \ldots \ldots \ldots \ldots$ & 101 & $9^{\prime} \dot{t}$ \\
\hline
\end{tabular}

TABLEAU 3

Composition des rigimes en aminoacides indispensables

\begin{tabular}{|c|c|c|c|c|c|c|}
\hline & \multirow{2}{*}{\multicolumn{2}{|c|}{$\begin{array}{c}\text { Régrime de base pour } \\
\text { les animaux de } 20 \text { à } 60 \mathrm{~kg}\end{array}$}} & \multicolumn{4}{|c|}{$\begin{array}{l}\text { Régime de base pour les animaux de } \\
\qquad 60 \text { à } 100 \mathrm{~kg}\end{array}$} \\
\hline & & & \multicolumn{2}{|c|}{ l'eneurs calculées } & \multicolumn{2}{|c|}{ Teneurs dosées } \\
\hline & $\begin{array}{l}\text { P. } 100 \text { de } \\
\text { l'aliment }\end{array}$ & $\begin{array}{c}\text { p. } 100 \text { des } \\
\text { matieres } \\
\text { azotées }\end{array}$ & $\begin{array}{l}\text { p. } 100 \text { de } \\
\text { l'aliment }\end{array}$ & $\begin{array}{c}\text { p. } 100 \text { des } \\
\text { matieres } \\
\text { azotées }\end{array}$ & $\begin{array}{c}\text { 1. } 100 \text { des } \\
\text { matieres } \\
\text { azotées }\end{array}$ & $\begin{array}{c}\text { 1. } 100 \text { des } \\
\text { valeurs } \\
\text { calculées }\end{array}$ \\
\hline Arrinine $\ldots \ldots \ldots \ldots$ & 1,06 & 6,0 & 0,90 & 5,9 & $1 ;, 0$ & 103 \\
\hline Ilistidine. & 0,35 & 2,0 & 0,30 & $\because, 0$ & $\because, 1$ & 105 \\
\hline Isoleucine & 0,70 & 4,0 & 0,59 & 3,9 & $:, 3,5$ & 89 \\
\hline Leucine ..... & 1,22 & 6,9 & 1,05 & $b, 9$ & 7,0 & 101 \\
\hline Lysine. & 0,81 & 4,5 & 0,63 & 4,15 & 1,05 & 98 \\
\hline Méthionine & $0,2^{\prime} \mathrm{t}$ & 1,4 & $0, \geq 0$ & $1,{ }^{\prime} \prime$ & 1,1 & 80 \\
\hline Cystine....... & 0,36 & 2,0 & $0,3 y^{\prime}$ & 2,2 & 1,7 & 78 \\
\hline Phénylalanine. & 0,79 & 1,4 & 0,70 & 4,6 & 4,55 & 100 \\
\hline Tyrosine .... & 0,57 & 3,2 & 0,49 & 3,2 & $:, 1$ & 97 \\
\hline Thréonine .... & 0,65 & 3,7 & 0,54 & 3,6 & $: 3, ;$ & 93 \\
\hline Tryptophane (1) & 0,21 & 1,2 & 0,16 & 1,1 & - & - \\
\hline Valine $\ldots \ldots \ldots \ldots \ldots$ & 0,92 & 5,5 & 0,85 & 5,5 & 4,7 & 85 \\
\hline Methionine + cystine & 0,60 & 3,1 & 0,54 & 3,5 & $\because, 8$ & 80 \\
\hline
\end{tabular}

(1) D'après OrR et WATT (1957).

Il y a un bon accord entre les calculs et les résultats de dosage pour la plupart des acides aminés. L'écart constaté pour la valine (I8 p. IOo) et l'isoleucine(I 2 p. IOo) peut avoir deux explications : 1'hydrolyse acide en 24 heures utilisée pour les dosages chromatographiques ne permet pas leur libération complète et les résultats des dosages microbiologiques sont souvent élevés pour ces deux acides aminés. Le chiffre obtenu par le calcul doit être assez voisin de la réalité dans le cas de l'isoleucine, tandis que la teneur en valine doit être intermédiaire entre les deux valeurs obtenues. Un écart important est également observé entre résultats de dosage et résultats de calcul dans le cas des acides aminés soufrés. Cet écart peut être dî̀ à la difficulté du dosage 
microbiologique de ces acides aminés (cystine notamment) et à la variabilité de la teneur en cystine de certains produits (orge en particulier). Les chiffres obtenus par le dosage de l'aliment sont certainement les plus valables: les calculs semblent donc surestimer les teneurs en acides aminés soufrés.

La comparaison des teneurs en acides aminés du régime témoin $\mathrm{A}$ avec celles des protéines d'œuf (résultats de dosage chromatographique obtenus dans ce laboratoire), considérées comme protéines de référence par Mitcher, et Block (I946) permet de mettre en évidence un certain nombre de déficits (tableau 4). Pour l'aliment I correspondant à la premiède période, les déficits les plus importants concernent les acides aminés soufrés $(-43)$ (méthionine : -58 ; cystine : -23 ) et la lysine $(-36)$. Si l'on tient compte de la surestimation probable des teneurs en aminoacides soufrés due au mode de calcul, leur déficit est plus accentué et serait supérieur à 50 p. Ioo. Le déficit primaire de l'aliment de la seconde périođe (60 à Ioo $\mathrm{kg}$ ) porte sur les acides aminés soufrés $(-52)$ et le déficit secondaire concerne la lysine $(-42)$.

\section{TABLEAU 4}

Pourcentage de déficit des aminoacides indispensables des régimes témoins par rapport aux protéines d'ouf

\begin{tabular}{|c|c|c|c|c|}
\hline & \multirow{3}{*}{$\begin{array}{c}\text { Teneurs en } \\
\text { aminoacides des } \\
\text { protéines d'cuf (1) }\end{array}$} & \multicolumn{3}{|c|}{ Pourcentage de déficit } \\
\hline & & \multicolumn{2}{|c|}{ D'apris les teneurs calculées } & \multirow{2}{*}{$\begin{array}{l}\text { D'après les teneurs } \\
\text { dosées dans } \\
\text { l'aliment II } \\
\text { (animaux de } 60 \\
\text { à } 100 \mathrm{~kg} \text { ) }\end{array}$} \\
\hline & & $\begin{array}{l}\text { dans l'aliment I } \\
\text { (animaux de } 20 \\
\text { à } 60 \mathrm{~kg} \text { ) }\end{array}$ & $\begin{array}{l}\text { dans l'aliment II } \\
\text { (animaux de } 60 \\
\text { à } 100 \mathrm{~kg})\end{array}$ & \\
\hline Arginine $\ldots \ldots \ldots \ldots \ldots$ & 6,4 & 7 & 8 & 6 \\
\hline Histidine ............ & 2,6 & 24 & 23 & 19 \\
\hline Isoleucine $\ldots \ldots \ldots \ldots$ & 5,5 & 28 & 29 & 37 \\
\hline Leucine $\ldots \ldots \ldots \ldots \ldots$ & 8,5 & 19 & 19 & 24 \\
\hline Lysine ............. & 7,1 & 36 & 42 & 43 \\
\hline Méthionine ........... & 3,3 & 56,5 & 61 & 67 \\
\hline Cystine ............... & 2,6 & 23 & 15 & 33,5 \\
\hline Phénylalanine .......... & 4,85 & 9 & 6 & 6 \\
\hline Tyrosine ............. & 3,80 & 16 & 15 & 18 \\
\hline Thréonine ............ & 4,80 & 24 & 26 & 31 \\
\hline Tryptophane ............. & 1,65 & 28 & 35 & - \\
\hline Valine ................ & 7,0 & 22 & 21 & 33 \\
\hline Méthionine + cystine $\ldots$ & 5,9 & 43 & 40 & 52 \\
\hline
\end{tabular}

(1) Résultats d'analyse chromatographique (PION, 1961), sauf en ce qui concerne le tryptophane (ORR et WATT, 1957).

Les données concernant le pourcentage de déficit des acides aminés soufrés par rapport aux protéines d'œuf dans les régimes B et $\mathrm{C}$ sont rapportées dans le Tableau 5 . L'addition de I, $5 \mathrm{p}$. Iooo de LL méthionine au régime de base de la période I réduit le déficit en acides aminés soufrés, si l'on tient compte de la surestimation de leurs teneurs, à un niveau qui correspond approximativement au niveau du facteur limitant secondaire ; cette même addition fait apparaître la lysine comme facteur limitant primaire pour l'aliment de la deuxième période. L'addition de $3 \mathrm{p}$. I ooo de DL méthionine au régime de base comble pratiquement le déficit de méthionine par rap- 
port aux protéines d'œuf, le déficit global en aminoacides soufrés étant lui-même d’importance minime. Les contrôles effectués sur les régimes $\mathrm{B}$ et $\mathrm{C}$ ont permis de retrouver quantitativement les doses ajoutées.

TABLEAU 5

Pourcentage de défucit des aminoacides soufrés dans les régimes $B$ et $C$

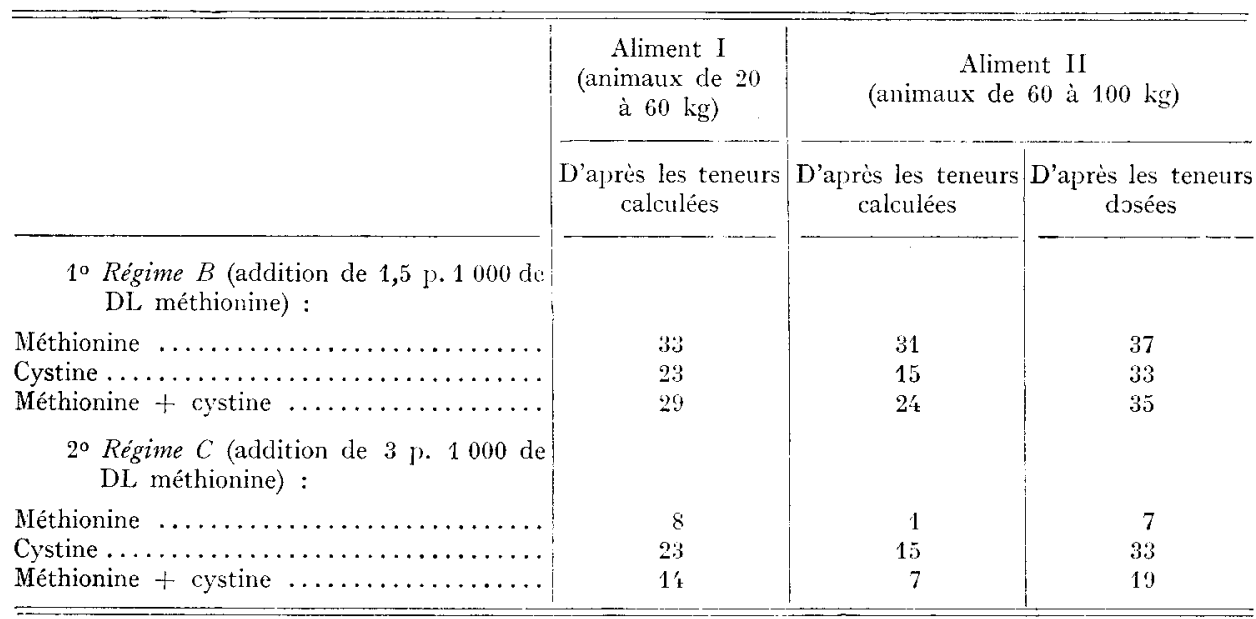

$2^{\circ}$ Croissance et consommation

En raison de la modification de composition de l'aliment lorsque les animaux atteignent $60 \mathrm{~kg}$ de poids vif, les données concernant la croissance et la consommation

TABI,EAU 6

Croissance et consommation (résultats moyens) pendant la période de croissance entre 20 et $60 \mathrm{~kg}$

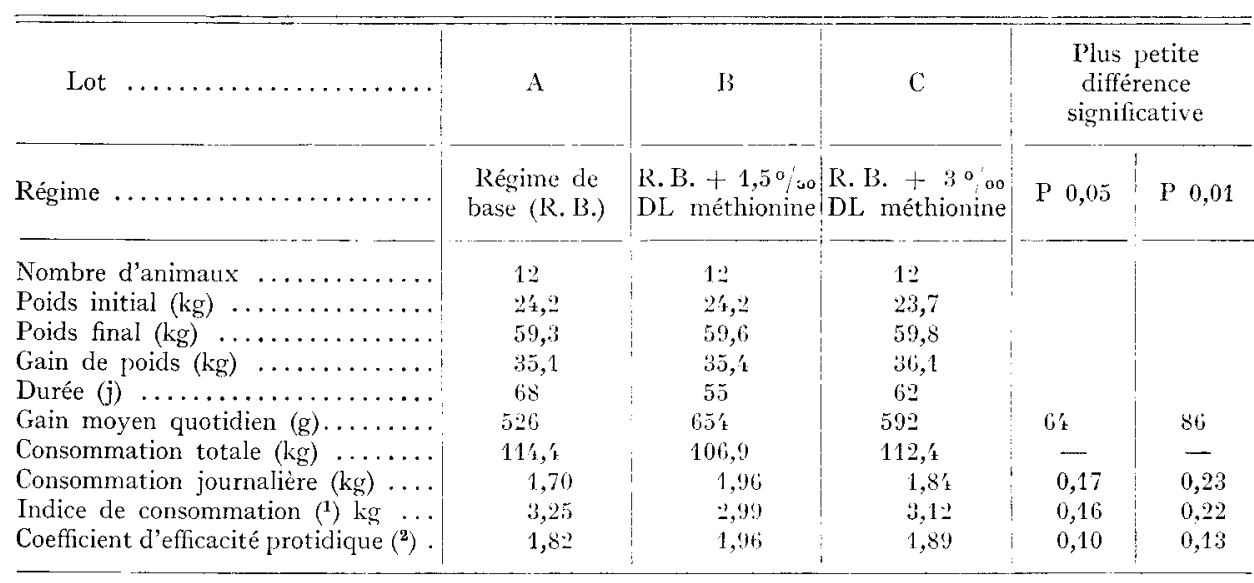

(1) Indice de consommation : $\frac{\text { quantité de nourriture ingérée }(\mathrm{kg})}{\text { gain cle poids }(\mathrm{kg})}$

$\left.{ }^{2}\right)$ Coefficient d'efficacité protidique $: \frac{\text { gain de poids }(\mathrm{kg})}{\text { quantité de protéines ingérées }(\mathrm{kg})}$ 
ont été divisées en deux périodes : elles figurent dans les tableaux 6 et 8 . I.es résultats concernant l'ensemble de la croissance sont rapportés dans le tableau 9 .

a) Croissance de 20 à $60 \mathrm{~kg}$ :

Entre 20 et $60 \mathrm{~kg}$ de poids vif (tableau 6), l'incorporation de DL méthionine au régime de base a des effets bénéfiques sur les performances des animaux.

L'addition de I,5 p. I ooo de DL méthionine a pour conséquence une amélioration hautement significative de la vitesse de croissance ( 24 p. Ioo par rapport au lot témoin), une augmentation hautement significative de la consommation ( $\mathrm{s}_{\mathbf{s}} \mathrm{p}$. Ioo) et une abaissement hautenent significatif de l'indice de consommation. C'est surtout au cours du premier mois d'expérience que l'action de la méthionine a été la plus marquée (tableau 7).

TABLEAU 7

Croissance durant les deux premiers mois

\begin{tabular}{|c|c|c|c|c|c|c|c|}
\hline \multirow[b]{2}{*}{ Lot } & \multicolumn{3}{|c|}{$0-28 \mathrm{j}$} & \multicolumn{4}{|c|}{$28-56 j$} \\
\hline & $\begin{array}{l}\text { Poids } \\
\text { initial } \\
(\mathrm{kg})\end{array}$ & $\begin{array}{l}\text { Poids } \\
\text { tinal } \\
(\mathrm{k} r)\end{array}$ & $\begin{array}{c}\text { Gain } \\
\text { moyen } \\
\text { quotidien }(g)\end{array}$ & $\begin{array}{l}\text { Poids } \\
\text { initial } \\
(\mathrm{kg})\end{array}$ & $!$ & $\begin{array}{l}\text { l'oids } \\
\text { final } \\
(\mathrm{kg})\end{array}$ & $\begin{array}{c}\text { Gain } \\
\text { moyen } \\
\text { quotidien }(g)\end{array}$ \\
\hline$A \ldots \ldots \ldots \ldots$ & 24,2 & $3 t, 6$ & $\begin{array}{l}371 \\
(100)(1)\end{array}$ & $3^{\prime},{ }^{\prime},{ }^{\prime}$ & & 50,8 & $\begin{array}{c}579 \\
(100)(\mathrm{l})\end{array}$ \\
\hline$B \ldots \ldots$ & 24,2 & 39,9 & $\begin{array}{l}5+11 \\
(151)(1)\end{array}$ & $: 39,9$ & i & 59,7 & $\begin{array}{l}707 \\
(122)(\mathbf{1})\end{array}$ \\
\hline $\mathrm{C} \ldots \ldots \ldots \ldots \ldots$ & $2: 3,7$ & 32,6 & $\begin{array}{l}496 \\
(13 i)(1)\end{array}$ & 37,6 & & $5 \tilde{3}, 1$ & $\begin{array}{l}6: 32 \\
(109)(1)\end{array}$ \\
\hline
\end{tabular}

(1) Les chiffres placés entre parenthèses indiquent les gains de poids relatifs des lots Is et $\mathrm{C}$ par rappont au témoin (100).

L'effet d'une addition de $3 \mathrm{p}$. I ooo de I)L méthionine est beaucoup moins sensible : si l'amélioration de croissance ( $\mathrm{r} 2 \mathrm{p}$. Ioo par rapport au lot témoin) est encore significative, l'augmentation de consommation journalière $(S \mathrm{p}$. Ioo) ne l'est plus; il en est de même pour l'abaissement de l'indice de consommation (4 p. Ioo).

Par rapport aux animaux recevant le régime contenant $I, 5 \mathrm{p}$. I ooo de $\mathrm{UL}$, méthionine, ceux recevant le régime à $3 \mathrm{p}$. Iooo de cet acide aminé présentent une croissance abaissée (de 9 p. Ioo) et une consommation dininuée.

\section{b) Croissance de bo à Ioo $\mathrm{kg}($ tableau s):}

Entre 60 et $100 \mathrm{~kg}$ de poids vif, les différences entre animaux des divers lots s'estompent ou s'annulent. La vitesse de croissance des animaux du lot B et leur consommation sont légèrement supérieures à celles des animaux des autres lots, mais la différence n'est pas significative. 


\section{TABIEAU 8}

Croissance et consommation (résultats moyens) pendant la période de croissance entre 60 et $100 \mathrm{~kg}$

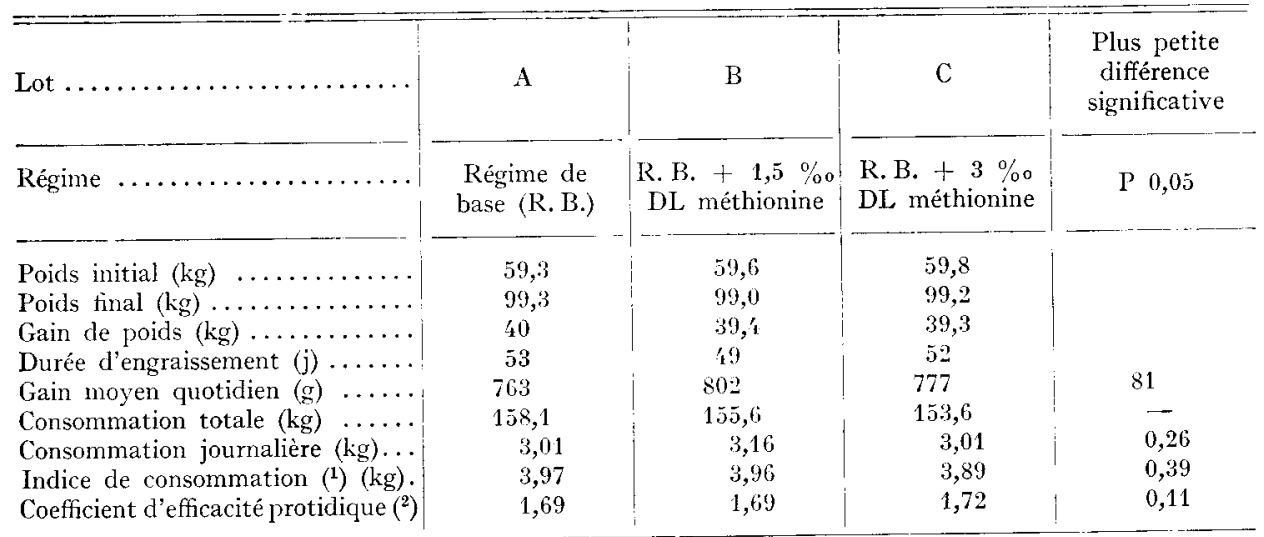

(1) Indice de consommation : $\frac{\text { quantité de nourriture ingérée }(\mathrm{kg})}{\text { gain de poids }(\mathrm{kg}) \text {. }}$

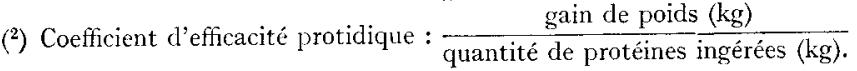

c) Croissance de 20 à Ioo $\mathrm{kg}$ :

L'addition de DL méthionine au régime de base a, dans les deux cas, eu des effets favorables sur les performances des animaux, l'amélioration la plus notable étant

TABIEAU 9

Résullats moyens concernant la croissance el la consommation (animaux de $20 \dot{a} 100 \mathrm{~kg}$ )

\begin{tabular}{|c|c|c|c|c|c|}
\hline Lot $\ldots \ldots \ldots \ldots \ldots \ldots \ldots \ldots$ & A & $\mathrm{B}$ & $\mathrm{C}$ & \multicolumn{2}{|c|}{$\begin{array}{l}\text { Plus petite } \\
\text { différence } \\
\text { significative }\end{array}$} \\
\hline Régime $\ldots \ldots \ldots \ldots \ldots$. & $\begin{array}{l}\text { Régrime de } \\
\text { base (R. B.) }\end{array}$ & $\mid \begin{array}{l}\mathrm{R}, \mathrm{B} .+1,5 \% \\
\mathrm{DI}, \text { méthionine }\end{array}$ & $\begin{array}{l}\text { R. B. }+3 \text { oo } \\
\text { DI méthionine }\end{array}$ & P 0,05 & P 0,01 \\
\hline Nombre d'animaux & 12 & 12 & 19 & & \\
\hline Poids initial $(\mathrm{kg}) \ldots$ & 21,2 & 24,2 & 23,7 & & \\
\hline Poids final $(\mathrm{kg}) \ldots \ldots \ldots \ldots \ldots$ & 99,3 & 99,0 & 99,2 & & \\
\hline Gain de poids $(\mathrm{kg}) \quad \ldots \ldots \ldots \ldots$ & 75,2 & $7 \prime, 2$ & 75,5 & & \\
\hline Durée d'engraissement (j) ...... & 121 & $10^{\prime}$ & 114 & & \\
\hline Gain moyen quotidien $(\mathrm{g}) \ldots \ldots \ldots$ & $62 \%$ & 719 & 673 & 47 & 63 \\
\hline Consommation totale $(\mathrm{kg}) \ldots \ldots$ & 272,5 & 262,5 & 266,1 & 一 & - \\
\hline Consommation journalière $(\mathrm{kg}) \ldots$ & 2,26 & 2,52 & 2,36 & 0,13 & 0,18 \\
\hline Indice de consommation $\left({ }^{1}\right) \ldots . .$. & 8,62 & 3,51 & 3,52 & 0,18 & 0,24 \\
\hline Coefficient d'efficacité protidique $\left({ }^{2}\right)$. & 1,74 & 1,81 & 1,79 & 0,08 & 0,11 \\
\hline
\end{tabular}

(1) Indice de consommation: $\frac{\text { quantité de nourriture ingérée }(\mathrm{kg})}{\text { gain de poids }(\mathrm{kg})}$

$\left.{ }^{2}\right)$ Coefficient d'efficacité proditique : $\frac{\text { gain de poids }(\mathrm{kg})}{\text { quantité de protéines ingérées }(\mathrm{kg})}$. 
enregistrée pour la plus faible dose administrée (lot $B$ ). La vitesse de croissance est accrue dans des proportions importantes ( 5 p. roo pour le lot B, 8 p. Ioo pour le lot C), les différences enregistrées étant significatives. L'ingestion journalière de nourriture est plus importante lorsque la méthionine est incorporée au régime (augmentation significative pour le lot B). L'indice de consommation n'est que faiblement amélioré par la présence de méthionine. Il en est de même du coefficient d'efficacité protidique.

\section{$3^{\circ}$ Composition CORPORELLE}

Les résultats concernant les mensurations effectuées sur les carcasses sont rapportés dans le tableau Io.

TABLEAU IO

Données relatives à la composition corporelle

\begin{tabular}{|c|c|c|c|}
\hline Lot $\ldots \ldots \ldots \ldots \ldots \ldots \ldots \ldots \ldots$ & A & $\mathrm{B}$ & $\mathrm{C}$ \\
\hline Régime $\ldots \ldots \ldots \ldots \ldots \ldots \ldots$ & $\begin{array}{l}\text { Régime de base } \\
\text { (R. B.) }\end{array}$ & $\begin{array}{l}\text { R. B. }+1,5 \% \\
\text { DI. méthionine }\end{array}$ & $\begin{array}{l}\text { R. B. }+3 \% \\
\text { DL méthionine }\end{array}$ \\
\hline Rendement $: \frac{\text { poids net }}{\text { poids vif }} \times 100 \ldots \ldots$ & 71,63 & 71,66 & 71,63 \\
\hline$\frac{\text { Jambon }+ \text { Longe }}{\text { poids net }} \times 100 \ldots \ldots \ldots$ & 50,11 & 50,40 & 50,38 \\
\hline$\frac{\text { Gras }+ \text { Panne }}{\text { poids net }} \times 100 \ldots \ldots \ldots \ldots$ & 19,55 & 19,40 & 19,16 \\
\hline Épaisseur de lard $\frac{(\text { rein }+ \text { dos })}{2}(\mathrm{~mm})$ & 28,6 & 29,1 & 30,0 \\
\hline
\end{tabular}

Bien que leur croissance ait été différente, les animaux présentent une composition corporelle invariable d'un lot à l'autre: le pourcentage de " morceaux maigres " (jambon + longe) semble légèrement plus élevé pour les animaux recevant de la méthionine dans leur régime, mais cette différence est loin d'être significative.

\section{DISCUSSION}

La méthode de MrTchelı, et Brock (I946), qui consiste à comparer les teneurs en aminoacides des protéines alimentaires avec celles des protéines d'œuf, considérées comme protéines de référence, présente une valeur prévisionnelle certaine, tant en ce qui concerne la valeur biologique de ces protéines que la nature et l'importance de leurs déficits en aminoacides. Mitchel, (I954) a, en effet, trouvé une relation très étroite $(r=0,83)$ entre la classe chimique des protéines déterminée par cette méthode et leur valeur biologique mesurée in vivo chez des animaux en croissance (rat, porc, 
chien). La difficulté de l'application de cette méthode réside dans le choix des valeurs à utiliser, tant pour la composition en acides aminés des aliments utilisés, que pour celle des protéines d'œuf. Il semblerait logique d'adopter les valeurs utilisées par MitcheiL, et Br,ock (I946) puisque ce sont elles qui ont permis à ces auteurs d'établir une relation entre composition en acides anninés et valeur biologique des protéines. Mais les matières premières utilisées dans nos aliments risquent d'ètre différentes des produits dont les analysses ont été utilisées par Mrtchel obstacle s'oppose à l'utilisation des diverses tables (ORR et WATT, I957 - WILLIAMs, I955, - Block et Weiss, I956- PFander et TribBi,E, I957). De plus, les méthodes analytiques ont évolué et les résultats obtenus par diverses méthodes ne sont pas toujours comparables. C'est pourquoi, dans la mesure du possible, les résultats d'analyse obtenus à Jouy-en-Josas (PIOx et de BEI.Suxce, Ig6I) ont servi de base pour les calculs. Toutefois, les produits introduits dans les régimes ne faisaient pas partie des mêmes lots que ceux sur lesquels avaient porté les analyses, ce qui explique en partie les écarts observés pour certains acides aminés entre les teneurs calculées et les teneurs dosées dans l'aliment II. Les pourcentages de déficit obtenus en comparant les valeurs obtenues par dosage de l'aliment Ir aux teneurs en aminoacides des protéines d'œuf, dosées dans le même laboratoire par la même méthode, devraient être les plus proches de la réalité. En ce qui concerne l'aliment I, on est en droit de penser que sa teneur en acides aminés soufrés est surestimée par le calcul puisque, dans l'aliment II, les chiffres obtenus par dosage sont inférieurs à ceux fournis par le calcul.

En outre, la teneur en cystine est relativement élevée, par rapport à la teneur en méthionine, dans les aliments utilisés et il est possible que les calculs effectués en utilisant la somme méthionine + cystine ne rendent pas exactement compte de la couverture du besoin spécifique en méthionine. Quoi qu'il en soit, les divers modes d'estimation permettent tous de conclure à un déficit en acides aminés soufrés. Seule l'importance de ce déficit est mal connue.

Sur le plan biologique, l'existence d'un déficit dans des protéines alimentaires. a des conséquences variables selon le taux d'introduction de ces protéines dans le régime, le problème de la qualité des protéines se ramenant à un problème de quantité ('TERronNe, I952). I a question se posait donc de savoir si le taux de protéines présentes dans notre régime de base (régime A) était suffisant ou non pour pallier les. effets biologiques dus au déficit primaire en aminoacides soufrés de ces protéines.

En première analyse, bien que ce régime soit conforme aux normes recommandées, son taux en acides aminés soufrés semble insuffisant puisque l'addition de méthionine se traduit par une amélioration de la croissance et de l'indice de consommation. L'accélération de la croissance et l'amélioration de l'indice de consommation (ou du coefficient d'efficacité protidique), lors de l'addition de doses croissantes d'un acide aminé à un régime, sont en effet les critères biologicues généralement adoptés pour montrer que cet acide aminé représente le facteur limitant primaire des protéines de ce régime et pour déterminer le niveau du déficit (RoseribrG, I959). Si l'on adopte ce point de vue, la méthionine serait le facteur limitant primaire du régime de base pour la période de 20 à $60 \mathrm{~kg}$, et la dose de méthionine synthéticuue à incorporer au régime pour ajuster son déficit à celui du facteur limitant secondaire se situerait approximativement à un niveau de $r, 5 \mathrm{~g} / \mathrm{kg}$ de régime. Pour la période de 60 à Ioo $\mathrm{kg}$, le déficit de méthionine serait beaucoup moins prononcé puisque 
aucune des deux doses ajoutées n'a provoqué d'amélioration significative des performances des animaux.

Cependant, certains points sont sujets à discussion : l'amélioration de la croissance et de l'indice de consommation est accompagnée d'une augmentation du niveau de consommation. Le gain de poids supplémentaire peut donc être justifié au moins partiellement par l'augmentation de la consommation journalière, et la baisse de l'indice de consommation par la diminution relative de la part du besoin d'entretien dans le besoin total. Ces deux critères ne permettent donc pas de conclure de façon formelle à une amélioration de la valeur biologique des protéines. Celle-ci ne pourrait être démontrée qu'à l'aide de techniques permettant de mesurer la rétention azotée chez des animaux consommant la même quantité d'aliment (technique " pairedfeeding " de Mitchel, en cage à métabolisme). Toutefois, la qualité des protéines est un des facteurs qui régissent le niveau de consommation (OSBORNE et al, rgIg -Rosenberg et Rohdenburg, I952 - Albanese, I959). Fin outre, Abraham et al. (I96I) ont montré, dans des expériences de libre consommation énergétique en présence de quantités constantes de protéines de qualité variable, que lorsque la couverture du besoin azoté n'était pas pleinement assurée, la qualité et la quantité des protéines administrées au rat et au poulet avaient une influence marquée sur le niveau de consommation énergétique ; celui-ci est supérieur si les protéines ingérées sont de bonne qualité. L'augmentation constatée du niveau de consommation peut donc être, elle aussi, une conséquence de l'amélioration de l'équilibre en acides aminés du régime.

Si les résultats obtenus semblent montrer que la méthionine est le facteur limitant primaire des protéines du régime de base utilisé, ils ne permettent pas d'en chiffrer le déficit avec précision. L,e taux de supplémentation optimum est certainement inférieur à $3 \mathrm{~g} / \mathrm{kg}$ de régime, et probablement proche de $\mathrm{I}, 5 \mathrm{~g} / \mathrm{kg}$; mais il est possible que d'autres taux auraient entrainé une amélioration plus marquée.

Le besoin en acides aminés soufrés ne peut être évalué qu'approximativement à partir de cette expérience, compte tenu de l'imprécision de l'estimation de la tenenr en ces acides aminés dans les régimes témoins, et de la dose optimum de méthionine nécessaire pour combler le déficit. Ce besoin se situe entre 0,6 et 0,7 p. Ioo d'un régime à I U. F. $/ \mathrm{kg}$ pour la période de croissance de 20 à $60 \mathrm{~kg}$, et entre 0,4 et $0,5 \mathrm{p}$. Ioo d'un régime à $0,94 \mathrm{U} . \mathrm{F} / \mathrm{kg}$ pour la période de croissance de 60 à roo $\mathrm{kg}$. Aucune conclusion ne peut être tirée sur la part relative de chacun des denx aminoacides dans la couverture du besoin global en acides aminés soufrés.

De toute façon, la valeur obtenue dépend de la façon dont sont couverts les besoins pour les autres acides aminés : elle représente le taux nécessaire à la réalisation de la croissance autorisée par les autres constituants de la ration et en particulier par les autres acides aminés indispensables présents dans le régime. La dose de méthionine permettant la meilleure croissance correspond en fait à la quantité nécessaire pour ramener son déficit au niveau du limitant secondaire. Ceci explique pourquoi le besoin en acides aminés soufrés, tel qu'il a été précisé par Sher,Ton et al. (I95I), par CURTrN et al. (I952 a) et par nous-mêmes, est supérieur aux estimations de divers auteurs (BECKER et al., I955 - BEL, et al., I955) dont les animaux, à poids égal, ont présenté une croissance inférieure à celle que nous avons enregistrée. Toutefois, cette explication n'est pas valable pour rendre compte de l'écart entre nos valeurs et celles précisées par d'autres auteurs (ACKER et al., I959- BECKER et 
$a l$, I954 $a$, r954 $b$ - CATRox et $a l$, I953-EVANS, I958, I960 $a$, x960 $b$ - MEADE I956 $a$, I956 $b$ - PFANDER et TRIBBL,E, I957). Le désaccord peut s'expliquer ici par 1e fait que les régimes expérimentaux utilisés par ces auteurs contiennent des antibiotiques qui modifient le métabolisme de la flore intestinale et peuvent ainsi protéger les acides aminés ingérés d'une désamination partielle (FraNçors et MicheL, I958), ce qui entraîne une action d'épargne vis-à-vis de l'azote du régime (FÉVRIER et DUMONT, I957 - RERAT, I96I). Le besoin en acides aminés est donc probablement plus élevé pour des animaux ne recevant pas d'antibiotiques. Les valeurs que nous avons données sont donc essentiellement valables pour des animaux à haute vitesse de croissance et ne recevant pas d'antibiotique.

L'addition de $3 \mathrm{p}$. I ooo de DL méthionine au régime de base provoque des performances moins bonnes chez les animaux recevant ce régime que chez ceux dont le régime est additionné de I,5 p. I ooo de DL méthionine. Cette dose de méthionine se comporte donc comme une source de déséquilibre. La notion de déséquilibre par excès, initialement formulée par JACQUoT (r952), est maintenant devenue classique (HARPER, 1956), et de nombreux travaux ont mis en évidence de tels déséquilibres chez le rat, la souris et les volailles. En ce qui concerne le Porc, il ressort des expériences de ClAUSEN (I960-I96I) qu'un excès de méthionine se traduit par une dépression de la vitesse de croissance ; ces résultats sont donc en accord avec les nôtres, mais aucune explication satisfaisante de ce phénomène n'a été apportée.

Le fait que la composition corporelle n'ait pas été modifiée de façon significative par l'introduction de méthionine dans le régime de base est intéressant : il montre que la vitesse de croissance n'est pas le seul facteur susceptible de modifier l'adiposité d'une carcasse. Dans ce cas précis, on peut penser que la rétention azotée journalière a été augmentée grâce à l'introduction de méthionine dans le régime, le dépôt supplémentaire d'azote étant dû aussi bien à l'amélioration de la valeur biologique des protéines du régime par la méthionine, qu'à l'augmentation de consommation azotée. Parallèlement, s'est produite une augmentation de la rétention calorique journalière, liée à l'augmentation de la consommation calorique.

\section{Rę̧u pour publication en juillet 1962}

\section{SUMMARY}

SUPPLEMENTATION BY DL METHIONINE OF A COMPLEX DIET FOR GROWING FINISHING PIGS

The present experiment was carried in order to know if a complex diet, which meets the recom. manded standards for nitrogen level, is able to provide the specific requirements for aminoacids of growing finishing pigs. For pigs weighing between twenty and sixty kilograms, the basal diet was made of barley ( 78 p. I00), peanut oil meal (4 p. 100), soy bean oil meal (4 p. I00), alfalfa meal (3 p. I00), distillers'yeast (4 p. 100), herring meal (2 p. I00), meat meal ( 2 p. I00), mineral and vitamin mixture ( $3 \mathrm{p} . \mathrm{I} 00)$; it contained I $7 \mathrm{p}$. 100 crude protein, $0,8 \mathrm{p}$. I00 lysine, 0,5 to $0,6 \mathrm{p}$. I00 sulfuraminoacids. For pigs above sixty kilograms, it was slightly modified in order to supply I 5 p. 100 crude protein.

Thirty six Large White pigs, divided on the basis of ancestry, weight and sex were alloted to three treatments :

- treatment $\mathrm{A}$ : basal diet;

- treatment B : basal diet + r,5 p. I ooo DL methionine ;

- treatment $\mathrm{C}$ : basal diet + 3 p. I 000 DL methionine. 
They were individually fed. Feeding was semi ad libitum.

The addition of I, 5 p. I 000 DL methionine to the basal diet improved rate of growth (24 p. Ioo) and food conversion ( 8 p. Ioo) below the weight of sixty kilograms. The facts may be explained by higher consumption level and a better balance between aminoacids. For pigs heavier than sixty kilograms, the improvement was not significant.

The addition of $3 \mathrm{p}$. I $000 \mathrm{DL}$ methionine improves but slightly performances of pigs, which can be attributed to aminoacid imbalance. Since sulfuraminoacids are limiting factors of the diet, the level of the needs for these amino acids can be calculated. Their optimum level for high rate of growth is about 0,6 to $0,7 \mathrm{p}$. I 00 of the diet for pigs between twenty to sixty kilograms, and between 0,4 to 0,5 p. roo of the diet for pigs heavier than sixty kilograms.

\section{RÉFÉRENCES BIBLIOGRAPHIQUES}

Abraham J., Calet C., Rerat A., Jacquot R., ig6r. Solidarité des besoins énergétique et protéique de croissance ; l'ajustement spontané des calories et des protides. C. R. Acad. Sci., 253, 2768-2770.

Acker D. C., Catron D. V., HaYs V.W., 1959. Lysine and methionine supplementation of corn-soybean oil meal rations for pigs in drylot. J. Anim. Sci., 18, $1053-1058$.

Albanese A. A., 1959. Criteria of protein nutrition in Albanese A. A., Protein and anino acid nutrition, 297-347. Acad. Press Ed. N. X. and London.

ALmoUist H. J., I959. The amino acid requirements of swine in AlBANEsE A. A., Protein and amino acil nutrition, 349-380. Acad. Press Ed. N. Y. and London.

Becker D. E., Ullrey D. E., Terrill S. W., I954 a. Protein and amino acid intakes for optimum growth rate in the young pig. J. Anim. Sci., 13, 346-356.

Becker D. E., Lassiter J. W., Terrilt. S. W., Norton H. W., r954 b. Levels of protein in practical rations for the pig. J.Anim. Sci., 13, 6r I-62r.

Becker D. E., Jensen A. M., Terrild. S. W., Norton H. W., I955. The methionine-cystine need of the young pig. J. Anim. Sci., 14, I086-1094.

Bell J. M., Williams H. H., Loosli J. K., Maynard L. A., i95o. The effect of methionine supplementation of a soybean oil meal-purified ration for growing pigs. J. Nutr., 40, 55 I-56r.

Block R. J., WeIss K. W., I956. Amino acid handbook. Methods and results of protein analysis. C. C. THOMAS, Spring field, Illinois.

Catron D. V., Acker D. C., Ashton G. C., Maddock H. M., Speer V. C., i953. Lysine and/or methionine supplementation of corn-soybean oil meal rations for pigs in drylot. J. Anim. Sci., 12 gro.

Clatsen H., I96r. Rapport concernant les expériences de reproduction et d'alimentation sur porcs réalisées par le laboratoire de recherches sur l'économie nurale et les abattoirs coopératifs danois (en danois). Frederiksberg Bogtrykkeri, Copenhague.

Curtin L. V., Loosli J. K., Willman J. P., Williams H. H., I952 a. Methionine as a supplement to soybean oil meal for weanling pigs. J. Animal Sci., 11, 459-464.

Curtin L. V., Loosli J. K., Abraham J., Williams H. H., Maysard L. A., 1952 b. The methionine requirement for the growth of swine. J. Nutr., 48, 499-508.

EAstoe J. E., LoNg J. E., I960. The amino acid composition of processed bones and meat. J. Sci. Food Agric., 11, 87-92.

Evans R. E., I958. Nutrition of the bacon pig. XIX. The requirement of the bacon pig for certain essential amino acids. J. Agric. Sci., 50, $230-242$.

Evans R. E., I960. The effect of adding lysine and methionine to the diet kept on low-protein vegetable foods. J.Agric. Sci., 54, 266-273.

Evans R. J., Bandemer S. L., Bauer D. H., rg6o. Cystine content of proteins, foods and feeds. Comparison of chromatography on a sulfonated polystyrene resin and microbiological methods of determination. $J$. Agric. Food. Chem., 8, 383-386.

Fevrier R., Dumont B. L., I957. Aureomycine et lipogenèse chez le Porc. I-Ration pauvre en protéines $4^{\mathrm{e}}$ Congrès International de Nutrition, Paris (résumé des communications, p. 78).

Françors A., Michel M., r958. Activité catabolique de la flore intestinale. Ann. Nutr. Paris, 13, I 52-і6г.

Harper A. E., 1956. Amino acid imbalances, toxicities and antagonisms. Nutr. Rev., 14, 225-227.

Horn M. J., Jones D. B., BLum A. E., 1946 a. Colorimetric determination of methionine in proteins and foods. J. Biol. Chem., 166, 3I3-320.

Horn M. J., Jones D. B., BLum A. E., I 946 b. Microbiological determination of methionine in proteins and foods. J. Biol. Chem., 166, $32 \mathrm{I}-326$.

Horn M. J., Jones D. B., Blum A. E., I947 a. Microbiological determination of lysine in proteins and foods. J. Biol. Chem., 169, 7I-76.

HoRn M. J., Jones D. B., BuUM A. E., 1947 b. Microbiological determination of threonine in proteins and foods. J. Biol. Chem., 169, $739^{-} 743$. 
Horn M. J., Joses D. B., BLux A. E., I947 ८. Yicrobiological determination of valine in proteins and foods. J. Biol. Chem., 170, 7 I9-723.

Horn M. J., Jones D. B., BLty A. E., I948 a. Nicrobiological determination of histidine in proteins and foods. J. Biol. Chem., 170, 149-I 54.

Horn M. J., Jones D. B., Blem A. E., I 948 b. Microbiological determination of arginine in proteins and foods. J. Biol. Chem., 176, 59-63.

Horn M. J., Jones D. B., Blum A. E., I948 c. Microbiological determination of phenylalanine in proteins and foods. J. Biol. Chem., 176, 679-684.

Horn M. J., Jones D. B., BLUm A. E., I949a. Microbiological determination of leucine in proteins and foods. J. Biol. Chem., 177, 697. 701.

HORN M. T., JoNEs D. B., BuUM A. E., r949 b. Microbiological determination of isoleucine in proteins and foods. J. Biol. Chem., 180, 695-701.

Hunter W. L., I948. Report on crude fiber. J. Ass. Off. Agric. Chem. Wash., 31, 6I6.

JACQuot R., 1952. Premiers cours pour la formation des nutritionistes pour l'Afrique au sud du Sahara (O. A. A.jO. M. S.), Narseille.

Lison L., 1958. Statistique appliquée a la biologie expérimentale. La planification de l'expérience et l'analyse des résultats. Gauthier-Villars, Paris.

Lyman C. M., Kunken K. A., Hale F., 1956. Amino acids in feedstuffs ; content of farm feeds. J. Agric. Food Chem, 4, I008-1013.

I.yman C. M., Came B. J., Tiller H. M., I958. Amino acids in feedstuffs. The tyrosine content of farm feeds. J. Agric. Food Chem., 6, 767-769.

MEADE R. J., I956 a. The influence of tryptophan, methionine and lysine supplementation of a corn-soybean oil meal diet on nitrogen balance of growing swine. J. Anim. Sci., 15, 288-296.

MEAde R. J., 1956 b. The influence of methionine supplementation of I 2,14 and I6 percent protein cornsoybean oil meal diets upon nitrogen balance of growing swine. J. Nutr., 60, 599-608.

Mitchell H. M., Block R. J., 1946. Some relationships between the amino acid contents of proteins and their nutritive values for the rat. J. Biol. Chem., 163, 599-620.

Mitchell H. M., I954. Evaluation of protein adequacy. Biological value of proteins and amino acid interrelation in "Methods for Evaluation of Nutritional Adequacy and Status". Washington D. C., National Academy of Science-National Research Council, Advisory Board on Quarter master Research and Development, Comittee on foods, p. $3^{\mathrm{I}} 3$.

MoOre S., SteIN W. H., 1954. A modified ninhydrin reagent for the photometric determination of amino acids and related compounds. J. Biol. Chem., 211, 907-913.

Mooke S., Spackmay D. H., Steix W. H., 195. Chromatography of amino acids on sulfonated polystyrene resins. An improved system. Analy?. Chem., 30, I I85-IIgo.

1959. Nutr. Req. of Swine. Nutr. Req. of dom. anim. No 2. Rept. Com. Animal Nutr. Nat. Res. Coun. publ. 648. Nat. Acad. Sci. U.S. A.

ORR M. L., WATt B. K., I957. Amino acid content of foods. Home economics Research, Report 4, U. S. D. A., Washington.

Osborne R. B., Meñel L. B., Ferry E. L., rgig. A method of expressing numerically the growth promoting value of proteins. J. Biol. Chem., $3 \%, 223$.

Pfander W. H., Tribble L. F., r957. Amino acid composition of swine rations and amino acid requirements of weanling pigs. Bull. Mo. Agric. Exp. Sia., Bulletin 626.

Pion R., Bessunce (de) C., ig6r. (Communication personnelle.)

Rerat A., r961. Les antibiotiques en alimentation animale. Ann. Nutr. Paris., 15, г 32-г40.

Rohdenburg E. L., Rosenberg H. R., I956. Estimation and stability of added DL, Methionine in mixed feeds. J. Agric. Food Chem., 4, 872-874.

Rosenberg II. R., 1959. Amino acid supplementation of foods and feeds in Albanese A. A, Protein and amino acid nutrition. Acad. Press ed. $\mathrm{N}$. Y. and London, 38I-4I 7 .

ROSEnberg H. R., RohdenbURG E. I.., r952. The fortification of bread with lysine. 2 . The nutritional value of fortified bread. Arch. Biochem. Biophys., 37, 46r-468.

Sirelton D. C., Beeson W. M., Mertz E. T., I95I. The effect of Methionine and Cystine on the growth of weanling pigs. J. Anim. Sci., 10, 57-64.

SNEDECOR G. W., 196r. Statistical methods applied to experiments in agriculture and biology. 5 th ed., Iowa State Univ. Press.

Stokes J. L., Gunness M., Dwyer I. M., CAswell M. C., I945. Microbiological methods for the determination tion of amino acids. II An uniform assay for the ten essential amino acids. J. Biol. Chem., 160, 35-49.

Terronne E. F., I952. La synthèse protéique. C. N. R. S., ed. Paris, p. 539.

Tiuullier G., Falconneau G., de Prailaune S., Chevillard I., Rocile J., 1954. Dosage de la méthionine dans les produits végétaux employés dans l'alimentation animale. Ann. Zoolech., 3, $29-45$.

Van Entwistie P., Hunter W. L., 1949. Report on crude fiber. J. Ass. Off. Agric. Chem. Wash., 32, 65 I-656.

Williams H. H., Curtin L. V., Abraham J., Loosli J., Maynaro L. A., i954. Estimation of growth requirements for amino acids by assay of the carcass. J. Biol. Chem., 208, 277-286.

Willians H. H., 1955. Essential amino acids content of animal feeds. Mem. Cornell Agric. Exp. Stat., bull. 337 